\title{
Automatización de procesos en la administración de energía eléctrica en el área residencial
}

\author{
Víctor Nava \\ Universidad Privada Dr. Rafael \\ Belloso Chacin URBE vic.n78@gmail.com
}

\author{
Ricardo Fabelo \\ Universidad Privada Dr. Rafael \\ Belloso Chacin URBE \\ ingjuancrl@gmail.com
}

\author{
Juan Romero \\ Universidad Privada Dr. Rafael \\ Belloso Chacin URBE \\ rfabelo@urbe.edu.ve
}

(Tipo de Artículo: Investigación. Recibido el 05/03/2015. Aprobado el 17/07/2015)

Resumen. Esta investigación presenta el diseño e implementación de una "Automatización de Procesos en la Administración de Energía Eléctrica" (APAEE) en el área residencial. El sistema consiste en un software de administración de energía eléctrica que funciona en una computadora de control central que se conecta a través de una red inalámbrica bajo el estándar IEEE 802.15.4 y una topología en malla a dispositivos remotos encargados de controlar los servicios de iluminación y climatización en una residencia en función del consumo de electricidad y parámetros establecidos por los usuarios. El hardware está basado en microcontroladores AVR $\AA$ XMEGA $\circledast$ de la marca Atmel®. Un estudio en los patrones de consumo de electricidad en una residencia es llevado a cabo en el desarrollo de los algoritmos de control, por medio de la instalación de sensores que detectan presencia de personas y estado de conmutación tanto de lámparas como del ventilador y compresor en acondicionadores de aire. En base a los resultados obtenidos, el diseño y simulación de los algoritmos de control han de ser realizados en Matlab® para posteriormente ser implementados en el software de control. El desarrollo de la APAEE reducirá considerablemente el consumo de electricidad en el área residencial.

Palabras clave. Administración de energía eléctrica, automatización de procesos, IEEE 802.15.4, microcontrolador AVR® XMEGA® de $A$ tmel®, Matlab®, topología en malla.

\section{Electrical energy management process automation in the residential area}

\begin{abstract}
This research presents a design and implementation of an "Electrical Energy Management Process Automation" (EEMPA) in the residential area. The system consists of an electrical energy management software that runs on a central control computer connected through a wireless network under the IEEE 802.15.4 standard and a mesh topology, to remote devices responsible for controlling residential lighting and air conditioning services with respect to electricity consumption and user parameter settings. The hardware design is based on the AVR® XMEGA® series microcontroller from Atmel ${ }^{\circledR}$. For the control algorithms development, an electrical energy consumption pattern study is conducted by installing sensors for detecting the presence of people as well as lamps and air conditioning system fan motor and compressor on/off state changes, an analysis of the collected data is performed and based on its results, design and simulation of the control algorithms are conducted on Matlab® simulation tool in order to be implemented later in the control software. EEMPA development will considerably reduce the electrical energy consumption in the residential sector.
\end{abstract}

Keywords. AtmeI® AVR® XMEGA® microcontroller, electrical energy management, IEEE 802.15.4, Matlab $\AA$, mesh topology, Process automation.

\section{INTRODUCCIÓN}

La creciente demanda de energía eléctrica que se ha registrado en los últimos 10 años en el área residencial, exige la creación de soluciones que permitan el manejo eficiente de este recurso. En este sentido, una adecuada administración contribuye en gran medida al logro del objetivo antes mencionado. Asimismo, la automatización en la administración del consumo de energía eléctrica se presenta como una herramienta que releva a los usuarios de la tarea de administrar de forma manual el uso de la electricidad, y con seguridad permite lograr una reducción significativa de su gasto.

\section{PROBLEMÁTICA}

El consumo de energía eléctrica en el sector residencial ha estado creciendo en todas las regiones del mundo a un promedio de 3,4\% por año desde 1990. Parte de este crecimiento se debe a que hay más personas con acceso a la electricidad; sin embargo, en su mayoría se debe al incremento en el consumo de electricidad en cada vivienda [1].

Venezuela destaca como el consumidor de energía eléctrica más grande de América Latina, además es el país suramericano que más generación tiene por habitante y con el mayor consumo de electricidad en el área residencial. El crecimiento para el 2009 para el área comercial y residencial fue de $7 \%$ en 10 años. Sin embargo, para el 2010 se registró una proyección de crecimiento de 4,5\% comparado con el 2009. Para la fecha se observaron picos de consumo elevados registrados en casi todos los meses del año trayendo como consecuencia que las plantas térmicas e hidroeléctricas del sistema eléctrico nacional no pudieran mantener los niveles de demanda de potencia por carecer de suficiente capacidad de generación creando un déficit acumulado de 1600 megavatios [2]. Esto se debe en gran medida a los bajos costos que promueven la existencia de nuevos hábitos de consumo de electricidad; así como también a las conexiones ilegales al servicio de energía 
eléctrica, especialmente en el Estado Zulia en donde el consumo es tres veces más elevado que el consumo promedio de los demás estados y constituye una parte importante de la demanda de energía a nivel nacional. Por parte del proveedor de servicio está la obsolescencia de equipos en las centrales termoeléctricas e hidroeléctricas y la falta de mantenimiento e inversión en las infraestructuras, lo que da como resultado un sistema eléctrico altamente ineficiente [3].

Aun cuando los seres humanos pueden ser muy buenos controladores manuales debido a que poseen la capacidad de pensar y considerar muchas variables a la vez, la mayoría de las tareas de control son repetitivas; los humanos no son repetitivos, y en algunas ocasiones son olvidadizos. Debido a esto, utilizar un control automatizado en los procesos pertinentes a la administración de energía eléctrica agrega la ventaja de consistencia, capacidad de realizar operaciones repetitivas, y permite la observación de las operaciones del sistema [4].

\section{ANÁLISIS DE LOS RESULTADOS}

La presente investigación se desarrolló en tres (3) fases las cuales se presentan a continuación:

\subsection{Fase 1: Describir los procesos en la administración de energía eléctrica en el área residencial.}

En esta fase se pretende estudiar y determinar los procesos que conforman a la administración de energía eléctrica en el área residencial.

Los procesos presentes en la administración de energía eléctrica son: el monitoreo, registro, análisis y el control de forma que, la energía eléctrica sea utilizada con máxima eficiencia [5].

Por otra parte, en la referencia [6] se describen los procesos de una forma práctica y los encierran dentro de una técnica de análisis a la que llaman monitoreo de energía, determinación de metas y Reporte (por su nombre y siglas en inglés: "Energy Monitoring, Targeting and Reporting", MT\&R) y la definen como una técnica de gestión poderosa para establecer metas de reducción en el consumo de energía, controlar el desempeño energético y proyectar presupuestos de consumo a futuro.

Esta técnica ha probado ser efectiva en lograr ahorros en costos de energía como consecuencia directa de un monitoreo de desempeño efectivo y de crear la información de gestión necesaria para identificar e implementar medidas de eficiencia energética. Aún más, provee un marco de referencia para la verificación cuando medidas de ahorro energético son implementadas.

Las definiciones aplicadas a estos procesos son las siguientes:

- Monitoreo de energía: Es la recolección y análisis de información referente al uso de la energía. Su propósito es el establecer las bases para el control de gestión, determinar el cuándo y por qué el consumo de energía se desvía del patrón establecido, y proveer las bases para la toma de acciones de gestión donde sean necesarias.

- Determinación de metas: Es la identificación de los niveles deseados de consumo de energía hacia los cuales se desea trabajar como objetivo de gestión.

- Reporte: Cierra el lazo ubicando la información de gestión generada en una forma que permita un control continuo del uso de energía, alcanzar los objetivos de reducción y verificar el ahorro energético.

Fase 2: Relacionar esquemáticamente a las variables significativas de cada servicio.

En la presente fase se observan las dinámicas de los servicios seleccionados para el estudio, se describen los efectos que tienen las variables de los servicios sobre la variable del consumo eléctrico, se seleccionan las variables físicas para la eficiencia de los servicios y se realiza un diagrama esquemático para relacionar las variables de cada servicio.

Los servicios de una residencia seleccionados para esta investigación fueron los servicios de iluminación y de acondicionamiento de aire; los cuales según lo establecido en [7], se suelen administrar como sistemas separados.

En los sistemas de iluminación la variable que influye en el consumo de electricidad es la intensidad de luz. A mayor sean los requerimientos de iluminación, mayor es el consumo de electricidad. Para los sistemas de iluminación, la selección del tipo de lámpara o bombillo tiene un impacto directo en el consumo de energía eléctrica, entre más eficiente (mayor producción de luz por vatio) sea la lámpara, menor será la energía que se requiere para alcanzar la cantidad deseada de iluminación. Sin embargo, la selección del tipo de lámpara esta fuera del alcance de esta investigación.

Para los sistemas de acondicionamiento de aire, la variable que afecta al consumo de energía eléctrica es la temperatura tanto interna de la habitación como la externa del ambiente. El compresor exhibe un ciclo de trabajo que se divide en dos hemiciclos:

- Hemiciclo de encendido: Es el intervalo de tiempo durante el cual el compresor del acondicionador de aire se mantiene encendido.

- Hemiciclo de apagado: Es el intervalo de tiempo durante el cual el compresor del acondicionador de aire se mantiene apagado.

La temperatura externa interactúa con la temperatura interna a través de paredes, puertas (cuerpo y ranuras) y ventanas. Por esta razón entre mejor sea el aislamiento térmico, mayor será el control de temperatura en la habitación de interés y mientras mayor sea la temperatura ambiental, mayor será el tiempo del hemiciclo de encendido y menor el del hemiciclo de apagado. 
Escogidos los servicios se realizó el siguiente paso es la selección de las variables significativas para la automatización:

- Se hace selección de la intensidad de luz para incrementar la eficiencia en el servicio de iluminación.

- Para aumentar la eficiencia en el sistema de acondicionamiento de aire se selecciona el ciclo de trabajo del compresor. Esto es, la variación del tiempo en los hemiciclos de encendido y apagado.

- La presencia de personas en los ambientes es una variable que se selecciona en común para mejorar el desempeño de ambos sistemas, permitiendo el encendido de los servicios cuando estos sean necesarios y apagarlos cuando ya no sean requeridos.

En la Fig. 1 se puede observar la relación entre las variables significativas seleccionadas para el presente trabajo de investigación. Para el sistema de acondicionamiento de aire, la presencia determina si el equipo debe permanecer encendido o debe apagarse, el consumo de energía determina la variación en el ciclo de trabajo del compresor. En el servicio de iluminación la presencia determina si ésta debe ser encendida o apagada en algún ambiente determinado y el consumo de energía activa la regulación de la intensidad de luz.

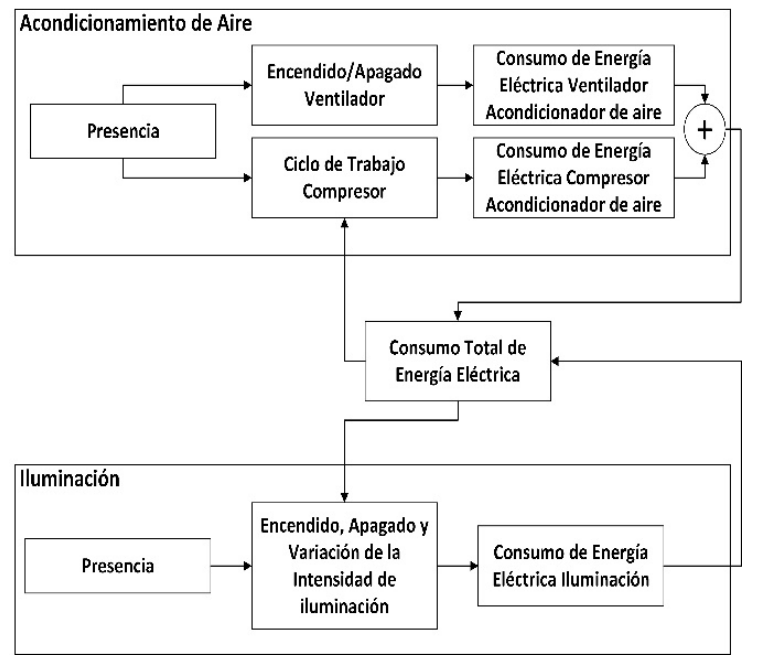

Fig. 1. Relación esquemática entre las variables de los servicios de una residencia

Para el sistema de iluminación se selecciona una estrategia de control compuesta de dos lazos cerrados regidos por una ecuación de control de cambio de nivel. En la referencia [8] se describe la ecuación de control de dos posiciones (ecuación 1).

$m=M_{1}$ cuando $e>0$

(1)

$m=M_{0}$ cuando $e<0$

En donde:

$m_{t}=$ Variable manipulada
$M_{1}$

= Valor máximo de la variable manipulada

$M_{0}$

= Valor mínimo de la variable manipulada

$e_{t}=$ Estado de la variable de control

En el primer lazo el control se basa en encender o apagar las lámparas en los ambientes dependiendo de si existe presencia o ausencia de personas. Por lo tanto se tiene que:

$m_{t}=$ Encendido/Apagado

$e_{t}=$ Presencia $/$ Ausencia

$M_{1}=$ Encendido

$M_{0}=$ Apagado

De donde se obtiene la ecuación (2).

$m=$ Encendido cuando $e=$ Presencia

(2)

$m=$ Apagado cuando $e=$ Ausencia

La tarea del segundo lazo de control es el de reducir la intensidad de iluminación a una intensidad mínima previamente configurada (set point) cuando el consumo de electricidad supera la meta de consumo preestablecida:

$m_{t}=$ Intencidad de luz

$e_{t}=$ Consumo de electricidad

$M_{1}=100 \%$

$M_{0}=$ Set point

Al reemplazar se obtiene la ecuación (3).

$m=100 \%$ cuando e $<$ Meta de consumo

(3)

$m=$ Setpoint cuando e $>$ Meta de consumo

Para aumentar las probabilidades de ahorro de energía, el lazo de control no incrementa la intensidad de iluminación si el consumo se encuentra por debajo de la meta. Esta acción se deja a discreción y comodidad del usuario. Si considera que el nivel de iluminación es muy bajo, éste, puede aumentar la intensidad de iluminación a un nivel más cómodo y con esto, establecer una nueva referencia de intensidad mínima para las iteraciones sucesivas.

Para el sistema de climatización la estrategia de control seleccionada para este servicio también está basada en dos lazos de control de dos posiciones. Al igual que en el sistema de iluminación, el primer lazo se encarga de controlar el encendido y apagado de la unidad de acondicionamiento de aire basándose en la presencia o ausencia de personas, como se ve en la ecuación (4). 
$m_{t}=$ Encendido $/$ Apagado

$e_{t}=$ Presencia $/$ Ausencia

$M_{1}=$ Encendido

$M_{0}=$ Apagado

La ecuación resultante es:

$m=$ Encendido cuando $e=$ Presencia

(4)

$m=$ Apagado cuando $e=$ Ausencia

Para el segundo lazo de control se emplea un valor de referencia (set point) dinámico que se ajusta dependiendo de si el consumo de electricidad se encuentra por debajo o por arriba de la meta de consumo. Éste lazo de control se encarga de manipular los valores de tiempo de ambos hemiciclos que componen el ciclo de trabajo del compresor, según ecuación (5).

$m e_{t}=$ Tiempo de encendido

$M e_{1}=$ Setpoint $_{-} e i$

$M e_{0}=$ Setpoint_es

$m a_{t}=$ Tiempo de apagado

$M a_{1}=$ Setpoint_as

$M a_{0}=$ Setpoint_ai

$e_{t}=$ Consumo de electricidad

Donde:

Setpoint_ei = Tiempo mínimo de encendido del compresor

Setpoint_es = Tiempo máximo de encendido del compresor

Setpoint_ai = Tiempo mínimo de apagado del compresor

Setpoint_as = Tiempo máximo de apagado del compresor

Debido a que los set points son dinámicos, éstos se determinan de la siguiente manera:

Para el hemiciclo de encendido se tiene:

te $=t e-k 1$ cuando $e>$ meta de consumo

$t e=t e+k 1$ cuando $e<$ meta de consumo

Para el hemiciclo de apagado, se tiene la ecuación (6).

$t a=t a+k 2$ cuando $e>$ meta de consumo

(6)

$t a=t a-k 2$ cuando $e<$ meta de consumo

En donde ta y te son los intervalos de tiempo de encendido y apagado respectivamente, y $k 1$ y $k 2$ son constantes de tiempo en segundos. Los valores máximos y mínimos que pueden alcanzar estos set points también están regidos por constantes de saturación, por lo tanto sus condiciones de cambio están expresadas mediante la ecuación (7) y (8).

Hemiciclo de encendido:

te $=t e-k 1$ cuando $t e>t e_{-}$min

(7)

$t e=t e+k 1$ cuando te $<t e_{-} \max$

Hemiciclo de apagado:

$t a=t a-k 2$ cuando $t a>t a \_m i n$

(8)

$t a=t a+k 2$ cuando ta $<t a \_$max

Las constantes te_min y te_max son los valores de tiempo mínimo y máximo del hemiciclo de encendido del compresor mientras que $t a \_m i n$ y $t a \_m i n$ son los valores de tiempo máximo y mínimo del hemiciclo de apagado.

Una vez determinados los valores de los intervalos de encendido y apagado de los set points, se tienen la ecuaciones (9) y (10).

Hemiciclo de encendido:

Setpoint_ei $=$ te cuando $e>$ meta de consumo

(9)

Setpoint_es $=$ te cuando $e<$ meta de consumo

Hemiciclo de apagado:

Setpoint_as $=$ ta cuando $e>$ meta de consumo

Setpoint_ai $=$ ta cuando $e<$ meta de consumo

Finalmente, las ecuaciones (11) y (12) son los controles resultantes para el sistema de climatización.

Hemiciclo de encendido:

me $=$ Setpoint_ei cuando $e>$ meta de consumo

me $=$ Setpoint_es cuando $e<$ meta de consumo

Hemiciclo de apagado:

$m a=$ Setpoint_as cuando $e>$ meta de consumo

(12)

ma = Setpoint_ai cuando e < meta de consumo

Los dispositivos de control poseen transductores inalámbricos que se conectan a una red bajo el protocolo Lightweight Mesh de Atmel, el cual se implementa bajo el estándar IEEE 802.25.4, posee una topología de malla y proporciona una conexión de datos a una tasa de transferencia de hasta $250 \mathrm{Kbps}$. Cada dispositivo de control se comporta como enrutador, de manera que tienen la capacidad de reenviar los mensajes provenientes de otros dispositivos y de esta forma ampliar 
el alcance y robustez de la red [9]. Los datos que cada dispositivo transmite son: el número de identificación, los valores de las variables medidas por cada uno de sus sensores y el estado de acción de control que estén ejecutando. El coordinador de enlace, es el encargado de formar la red inalámbrica y de dar formato de los datos que recibe de los distintos dispositivos para ser enviados al computador de control a través de un enlace de transmisión-recepción asíncrono universal (USART).

El computador de control central envía comandos a los dispositivos remotos de control a través del coordinador de enlace quien da formato a los datos que recibe por el puerto serial para ser enviados a través de la red inalámbrica. Los datos de control que envía el computador de control son: el número de identificación del dispositivo de destino, el tipo de comando y el valor correspondiente a dicho comando, ver Fig. 2.

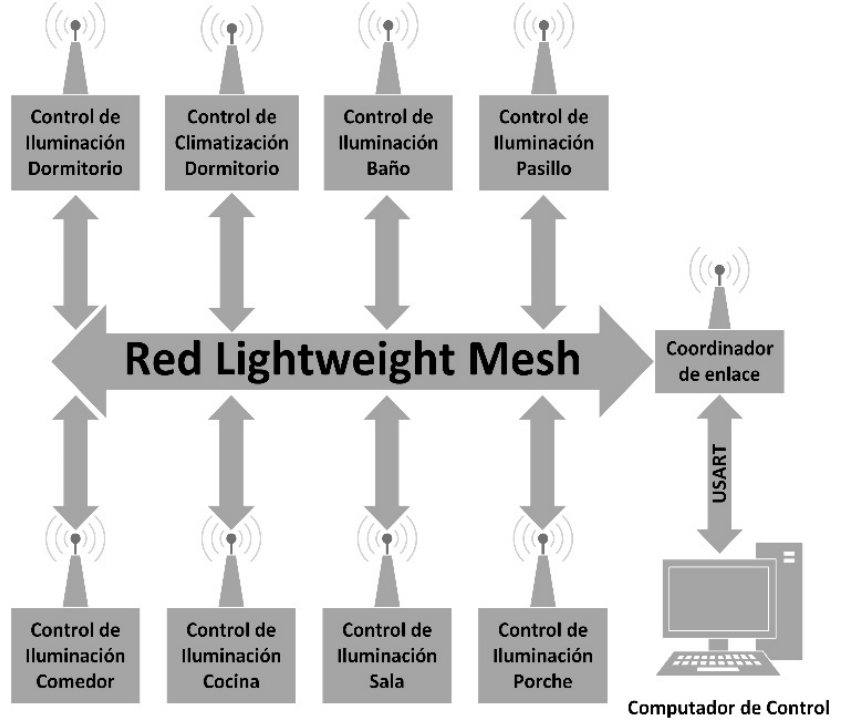

Fig. 2. Esquema de comunicación

La estrategia de automatización se basa en reducir el consumo de energía eléctrica en los hogares por medio del uso de dispositivos que se encargan de controlar niveles de iluminación, ciclos de trabajo de compresores en sistemas de acondicionamiento de aire, encendido y apagado de los servicios en respuesta a presencia o ausencia de personas y temporización en el caso de la iluminación externa. Los dispositivos de control envían valores de consumo de energía a través de la red de comunicación al computador de control el cual toma estos valores, analiza los datos, calcula las respuestas del sistema y las envía en forma de parámetros a través de la red a cada controlador remoto.

En los dispositivos de control se realiza la medición de energía en kilovatios-hora (KWh) que cada servicio consume por área; la medición de energía está dada por la ecuación (13).

$$
E=\int_{t 1}^{t 2} P_{(t)} d t
$$

Para llevar la energía al formato de KWh, se aplica la ecuación (14).

$$
K W h=\frac{E}{1000(3600 \text { seg })}
$$

Al sistema de gestión se le configura un valor de referencia (set point), el cual se refiere a la meta de consumo que se espera para un día determinado. Para mantener el consumo de energía eléctrica por debajo del nivel de referencia es necesario comparar constantemente el valor de consumo actual con la proyección del consumo basado en el set point. De manera que, el sistema pueda determinar si es necesario regular el consumo para no exceder la meta prestablecida. Para lograr esta tarea se compara la proporción que existe entre el consumo actual y el valor de consumo meta con la proporción entre el tiempo transcurrido y el tiempo total que corresponde a un día. Esto se expresa mediante las ecuaciones (15) a (17).

$$
\frac{\int_{t 1}^{t 2} P_{A 1(t)} d t+\int_{t 1}^{t 2} P_{A 2(t)} d t+\int_{t 1}^{t 2} P_{A n(t)} d t}{1000(3600 S e g) S P_{K W h}} \geq \frac{t}{t 2-t 1}
$$

Simplificando:

$$
\begin{aligned}
K W h_{a c t} & =\frac{\int_{t 1}^{t 2} P_{A 1(t)} d t+\int_{t 1}^{t 2} P_{A 2(t)} d t+\int_{t 1}^{t 2} P_{A n(t)} d t}{1000(3600 S e g)} \\
K W h_{a c t} & =K W h_{A 1}+K W h_{A 2}+K W h_{A n}
\end{aligned}
$$

En donde $K W h_{a c t}$ es el consumo actual total medido de todas las áreas automatizadas y el subíndice $A n$ se refiere a la cantidad de áreas presentes en el sistema.

Finalmente se obtiene la ecuación (18).

$$
\frac{K W h_{\text {act }}}{S P_{K W h}} \geq \frac{t_{\text {trans }}}{t_{\text {Tot }}}
$$

En donde:

$S P_{K W h}=$ Meta de consumo en kilovatios - hora

$t_{\text {trans }}=$ Tiempo transcurrido

$t_{\text {Tot }}=$ Tiempo total (86400seg) en un día

De esta forma, si el porcentaje de KWh que se han consumido es mayor al porcentaje de tiempo que ha transcurrido del día, el computador de gestión envía nuevos parámetros de funcionamientos a los dispositivos de control. Por ejemplo, al detectarse un exceso de consumo, el computador envía al controlador del servicio de climatización un nuevo parámetro de tiempo para el ciclo de trabajo del compresor aumentando el tiempo del hemiciclo de apagado y disminuyendo el tiempo del 
hemiciclo de encendido. A los controladores del servicio de iluminación, les envía parámetros para ajustar la intensidad de iluminación a niveles más bajos.

Una vez obtenidos los modelos de control se procede al diseño de los algoritmos. Para la presente investigación se utilizan dos plataformas, una basada en arquitectura x86-x64, bajo el sistema operativo Microsoft Windows y otra basada en microcontroladores AVR XMEGA de la marca Atmel.

Para la selección del sensor de voltaje y corriente se hizo referencia al diseño propuesto en la referencia [10] en la cual se describe un medidor de energía eléctrica basado en un microcontrolador XMEGA, pero en lugar de medir las señales de forma directa; se utiliza un circuito basado en amplificadores operacionales (OPAM) para condicionar las señales a valores que el microcontrolador pueda manejar. Con las mediciones del voltaje y la corriente se calculan los valores de potencia y energía.

Para la detección de movimiento se utilizó un sensor infrarrojo pasivo (PIR) comercial. Cuando el PIR detecta movimiento, la salida se activa proveyendo de un uno lógico con un voltaje de $3.3 \mathrm{~V}$, y el cual puede aprovecharse por un microcontrolador. El trabajo de este sensor es de dar aviso al microcontrolador de cuando hay presencia o ausencia de personas con el objetivo de activar los servicios solo cuando sean necesarios.

Los dispositivos de control están basados en microcontroladores AVR XMEGA de la marca Atmel. Para esta investigación, la medición de energía es de suma importancia y tal como se describe en [11], los microcontroladores AVR XMEGA tienen la capacidad hacer conversiones analógico/digitales en cuatro canales de forma simultánea, esto permite realizar un muestreo de corriente y voltaje al mismo tiempo, evitar desplazamientos entre las mediciones de las señales y por lo consiguiente, evitar la implementación de métodos de corrección de dichos desplazamientos.

Otra razón importante para la selección de este microcontrolador es el sistema de eventos. Este recurso único en los microcontroladores de Atmel, permite la transferencia de datos y el control de periféricos de forma independiente de la unidad central de procesamiento (CPU) o del sistema de interrupciones. Esto hace posible liberar a la CPU para realizar los cálculos de energía y que de forma simultánea se realicen nuevas conversiones analógico/digitales [12].

Para el control de iluminación se ha realizado un diseño similar al propuesto en [13], en la cual se manipula la intensidad de luz haciendo uso de un tríodo de corriente alterna (TRIAC) controlado por un microcontrolador. Sin embargo para el circuito usado en esta investigación, se realizaron algunas modificaciones al circuito propuesto. En este caso, el microcontrolador activa al TRIAC a través de un rectificador controlado de silicio (SCR), de esta forma se aísla galvánicamente el microcontrolador de la señal manipulada.

Para un correcto control de la iluminación es necesario sincronizar la señal de control con la frecuencia de la señal manipulada y para esto se hace uso de un sensor de cruce por cero, cada vez que la señal cruza por cero, éste sensor emite un pulso que le indica al microcontrolador que la señal está pasando por cero para que éste haga los procesos pertinentes a la manipulación adecuada y sincronizada de la señal. Para el control del acondicionador de aire se hizo uso de relés en el compresor y para apagar el sistema por completo.

A excepción de los actuadores, el hardware de control posee una estructura idéntica para ambos servicios. La Fig. 3 muestra un diagrama del diseño utilizado para el hardware de control de ambos servicios.

Cada dispositivo de control está compuesto de tres microcontroladores: un microcontrolador de campo que está conectado con los sensores y actuadores y se encarga tomar las mediciones analógicas y de generar las señales de control; un segundo microcontrolador se encarga de generar la interfaz gráfica del dispositivo, recibir comandos a través de pulsadores y sensores de presencia, enviar instrucciones de control y recibir datos de las mediciones del microcontrolador de campo a través de una interfaz USART, empaquetar los datos de mediciones y de acciones de control para ser enviados a través de la red inalámbrica e interpretar los comandos que provienen de la red para ser enviados y ejecutados por el microcontrolador (MCU) de nivel de campo. Un tercer microcontrolador se encarga de gestionar la transmisión y recepción de datos a través de la red inalámbrica.

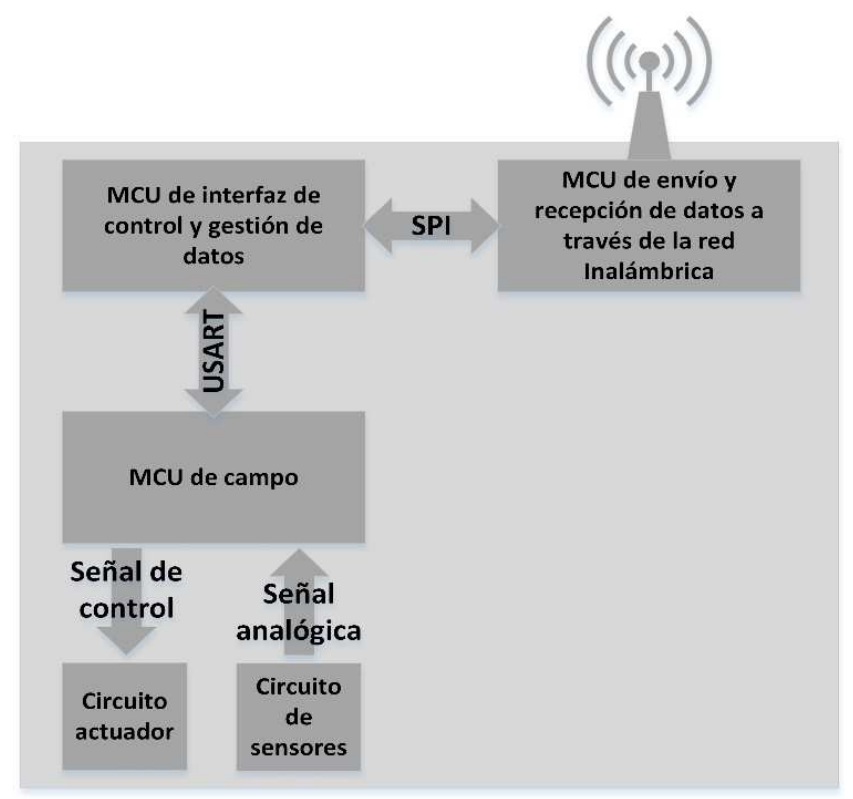

Fig. 3. Diagrama del hardware de control para los servicios de iluminación y climatización 
La Fig. 4 Muestra un diagrama del diseño del hardware supervisor, el cual está compuesto de un computador que ejecuta una aplicación de gestión de energía eléctrica conectado a un dispositivo coordinador de red.

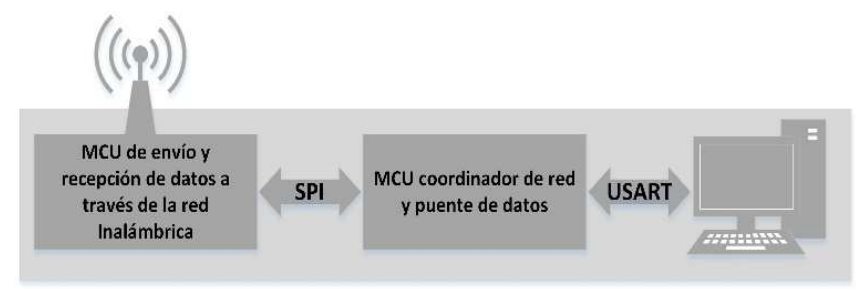

Fig. 4. Diagrama esquemático del hardware de supervisión

\subsection{Fase 3: Diseñar la automatización de los procesos en la administración de energía eléctrica en el área residencial.}

En esta última fase de realiza el diseño de los experimentos y se hacen pruebas comparativas en presencia y ausencia de automatización.

Para validar el funcionamiento de la automatización se realizaron dos experimentos, el primero está basado en una simulación realizada en la herramienta de análisis MATLAB, y el segundo experimento re realizó por medio de la implementación de la automatización en la habitación de una residencia para determinar si los resultados eran congruentes con los de la simulación y aumentaba el ahorro de energía eléctrica.

Para llevar a cabo la simulación se realizó una medición del comportamiento de consumo en una residencia durante siete días. La captura del comportamiento de consumo consistió en instalar sensores de presencia y sensores de cambio de estado (encendido y apagado) de lámparas, motor de ventilador y compresor en sistemas de acondicionamiento de aire. Estos sensores estaban conectados a un microcontrolador el cual se encargó de registrar la información de los sensores a intervalos de un segundo.

Se midieron un total de 15 variables:

- Presencia en dormitorio

- Estado de encendido/apagado de lámparas en dormitorio

- Estado de encendido/apagado de ventilador del acondicionador de aire en dormitorio

- Estado de encendido/apagado de compresor del acondicionador de aire en dormitorio

- Presencia en baño

- Estado de encendido/apagado de lámparas en baño

- Presencia en comedor

- Estado de encendido/apagado de lámparas en comedor

- Presencia en cocina

- Estado de encendido/apagado de lámparas en cocina

- Presencia en pasillo

- Estado de encendido/apagado de lámparas en pasillo
- Presencia en sala

- Estado de encendido/apagado de lámparas en sala

- Estado de encendido/apagado de lámparas en porche

Cabe destacar que debido a limitaciones de recursos, la medición de estas variables se realizó por partes. La primera semana se realizó el muestreo de las variables en el dormitorio y el baño, en una segunda semana se llevaron a cabo las mediciones en el comedor y la cocina y en una tercera semana se tomaron las muestras de las variables en el pasillo, la sala y el porche. Todas las mediciones comenzaron un lunes a las 12:00am y terminaron el domingo siguiente a las 11:59pm.

Una vez terminadas las mediciones, los datos se extrajeron de la memoria del microcontrolador y posteriormente guardados en un formato compatible con MATLAB. Las mediciones de las diferentes áreas se agruparon por día, esto es, todas las áreas medidas un día lunes se agrupan para representar el comportamiento de consumo de ese día; se repite el proceso para el resto de los días. Esto dio como resultado una matriz de comportamiento en la cual cada columna representa una variable medida, cada una con 86.400 muestras por día.

Para los valores de consumo de energía que se utilizaron en la simulación, se midió la potencia que consume un bombillo de 100 vatios. De igual forma se tomó la medición de la potencia consumida por el acondicionador de aire cuando el compresor estaba encendido y también cuando éste se encontraba apagado, de esta forma se obtuvo la potencia que consume tanto el compresor como el motor del ventilador.

Una vez obtenidos los valores de potencia y la matriz de comportamiento de consumo, éstos se ingresaron en una función realizada en MATLAB que toma como argumentos de entrada a la matriz de comportamiento y los valores de potencia y con ellos genera una nueva matriz de comportamiento, esta vez con los valores de consumo de energía eléctrica de cada lámpara y del ventilador y compresor del acondicionador de aire.

Con esta nueva matriz se pudo determinar el consumo total de cada día y que permitió establecer metas de consumo más bajas. Estas metas son los valores de referencia (set points) para la realización de la simulación.

Una vez determinados los set points, éstos se ingresan junto con la nueva matriz de comportamiento a una función realizada en MATLAB que posee los algoritmos de simulación de la automatización explicados en fases anteriores. Esta función arroja una nueva matriz de comportamiento, esta matriz expresa el comportamiento del consumo bajo las regulaciones de la automatización.

El segundo experimento consistió en la implementación de la automatización en los servicios de climatización e iluminación en el dormitorio de una residencia y está basada en los dispositivos descritos a lo largo de las fases anteriores. El objetivo es el de comprobar la congruencia entre el comportamiento que exhibe la automatización 
implementada, con el obtenido de las simulaciones realizadas en MATLAB y si se registra un ahorro significativo en el consumo de energía eléctrica.

Las pruebas de comparación realizadas se presentan en dos etapas, en la primera etapa se muestran graficas generadas de los datos de las simulaciones, las cuales se realizaron con tres valores de referencia diferentes: $20 \mathrm{KWh}$, 30KWh y 40KWh. Los resultados de estas simulaciones se compararon con el comportamiento de consumo de energía en ausencia de automatización.

En la Fig. 5 se observa la gráfica del consumo de del acondicionador de aire en el dormitorio de una residencia en el día dos del estudio. Se observa el consumo de energía en presencia y ausencia de automatización a distintos set points (SPs), las gráficas que la acompañan representan el comportamiento de la señal de control del compresor y el motor de ventilación para cada set point. La última gráfica de la serie representa la presencia de personas, la cual sirve para determinar el uso efectivo de la energía. La Fig. 6 muestra las gráficas de consumo del sistema de iluminación, el estado de conmutación e intensidad de luz en el dormitorio de nuevo, en presencia y ausencia de automatización a diferentes SPs. Por último, la gráfica de presencia de personas en el dormitorio.
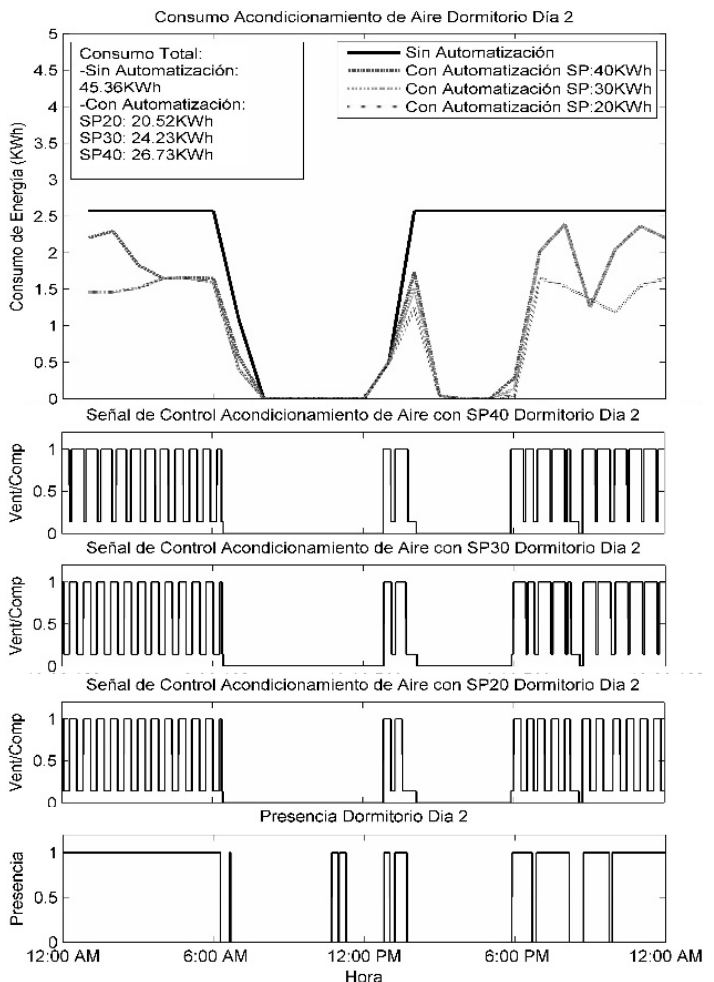

Fig. 5. Consumo de energía en acondicionador de aire en dormitorio en el día 2
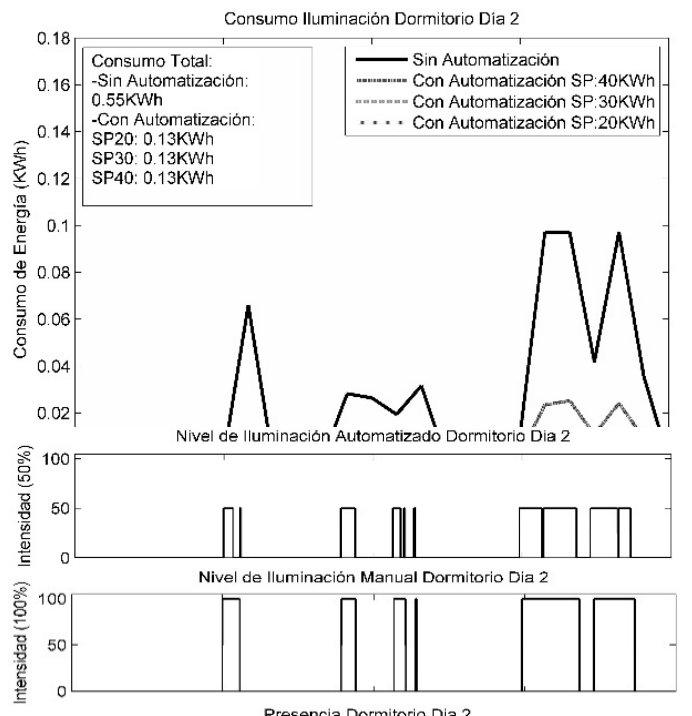

Presencia Dormitorio Dia

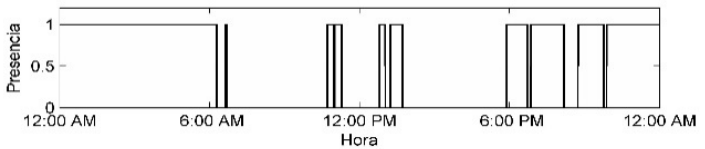

Fig. 6. Consumo de energía en acondicionador de aire en dormitorio en el día 2

En la segunda etapa de comparación se muestran gráficas de los datos que resultaron de implementar la automatización a los servicios de climatización e iluminación presentes en el dormitorio de la residencia seleccionada para este estudio. En la implementación sólo se hizo uso de un SP a $30 \mathrm{KWh}$. Las gráficas generadas se compararon con las del consumo promedio de energía eléctrica de cada servicio en dicho dormitorio durante los siete días registrados en la matriz de comportamiento previamente descrita.

La Fig. 7 muestra gráficas que representan la energía consumida por un equipo de acondicionador de aire en presencia y ausencia de automatización, la señal de control del ventilador y compresor, y la detección de presencia de personas. De igual forma, la Fig. 8 muestra la energía consumida por el servicio de iluminación en el mismo dormitorio en presencia y ausencia de automatización, seguida de las gráficas de la señal de control de iluminación y la de detección de presencia y ausencia de personas. 

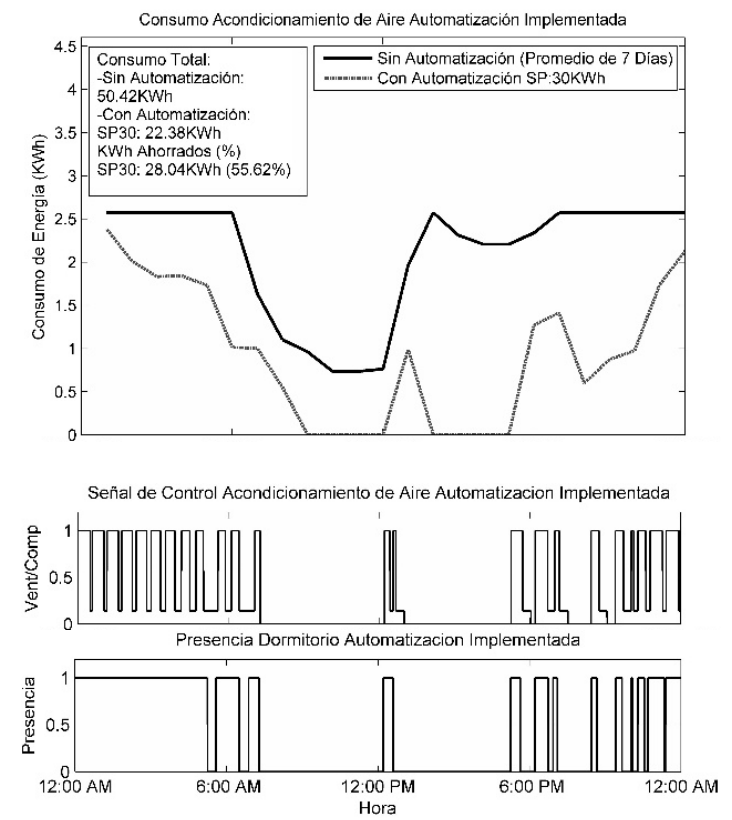

Fig. 7. Consumo del acondicionamiento de aire con automatización implementada
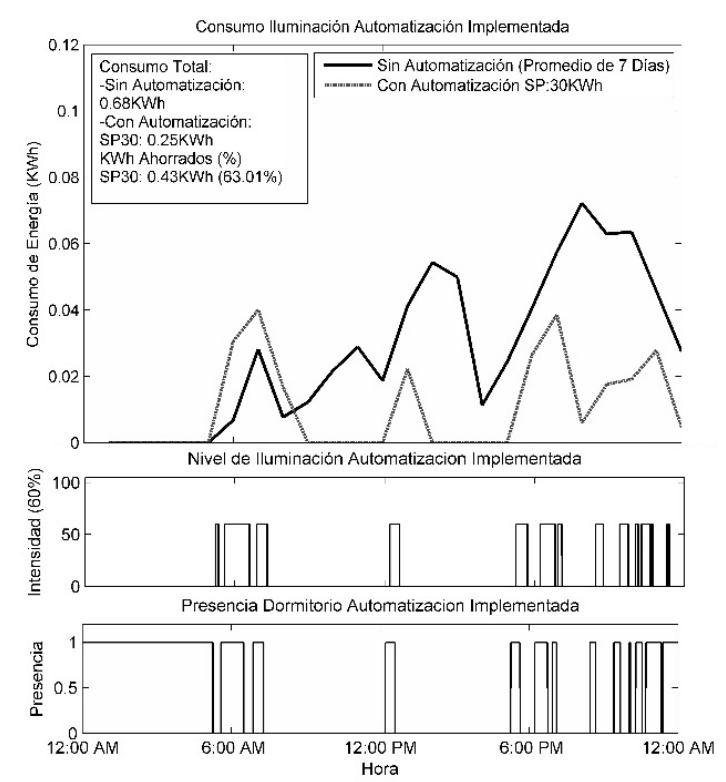

Fig. 8. Consumo del servicio de iluminación con automatización implementada

En la Fig. 9 se muestra la gráfica de las magnitudes del consumo de energía en ausencia y en presencia de automatización a diferentes SPs. En la Fig. 10 se observa la gráfica que representa el ahorro en magnitud y porcentual que propicia la implementación de la automatización a los set points establecidos. Ambas gráficas fueron generadas de la simulación de los algoritmos desarrollados para la presente investigación.

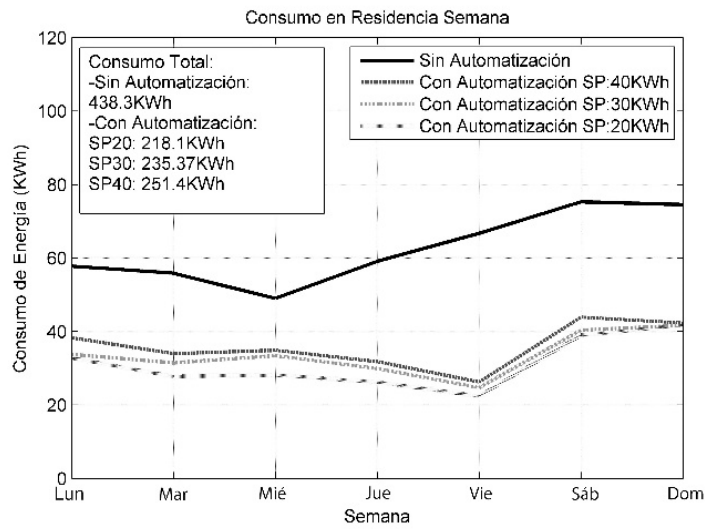

Fig. 9. Consumo total de electricidad en la semana

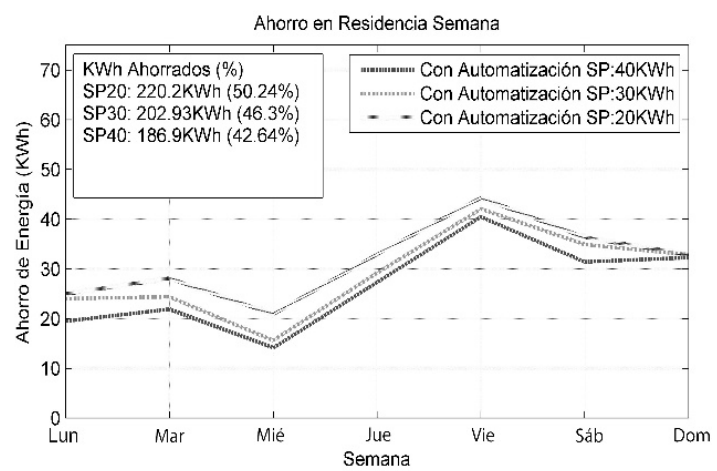

Fig. 10. Ahorro total de electricidad en la semana

El consumo de energía eléctrica en la residencia no se limita solamente a las áreas evaluadas. Según la información de facturación, el consumo promedio mensual de la residencia seleccionada para este estudio es de $4438 \mathrm{KWh}$. El consumo promedio diario de las áreas cubiertas por esta investigación en ausencia de automatización fue de $62.6 \mathrm{KWh}$, lo que equivale a $1878 \mathrm{KWh}$ en un periodo de 30 días. Por lo tanto, para calcular el porcentaje de ahorro verdadero que se obtiene de implementar la automatización con cada valor de SP, se usan las ecuaciones (19) a (24).

$K W h S P 20_{\text {prom }}=\left(\frac{218.1 K W h}{7}\right) * 30$ días $\cong 936 K W h$

$$
K W h S P 30_{\text {prom }}=\left(\frac{235.37 K W h}{7}\right) * 30 \text { días } \cong 1009 K W h
$$
(21)

$$
K W h S P 40_{\text {prom }}=\left(\frac{251.4 K W h}{7}\right) * 30 \text { días } \cong 1077 K W h
$$

$$
\begin{aligned}
& \text { AhorroSP20 }=\frac{1878 K W h-936 K W h}{4438 K W h} * 100=21.22 \% \\
& \text { AhorroSP30 }=\frac{1878 K W h-1009 K W h}{4438 K W h} * 100=19.60 \%
\end{aligned}
$$

$$
\text { AhorroSP40 }=\frac{1878 K W h-1077 K W h}{4438 K W h} * 100=18.04 \%
$$




\section{CONCLUSIONES}

Luego de realizado el trabajo de investigación y haber analizado la información resultante de los experimentos, se concluye que si es posible obtener un ahorro significativo del consumo de electricidad en el área residencial por medio de la implementación de una automatización en los procesos de administración de energía eléctrica.

Se pudo observar que en muchos casos, en ausencia de automatización, la energía eléctrica se utilizó de forma ineficiente. Al comparar las gráficas de presencia con las de consumo, se pudo identificar tiempos en los que se registró ausencia de personas por largos periodos mientras los servicios estaban encendidos. También se llegó a la conclusión de que la implementación de la automatización hizo uso de esos periodos de ausencia para reducir el consumo energético.

De la simulación se pudo concluir que en el transcurso de la semana, en las áreas evaluadas se obtuvo una disminución del consumo de energía eléctrica que representa un ahorro de hasta el $50.24 \%$ para un set point (SP) de $20 \mathrm{KWh}$, seguido de $46.3 \%$ y $42.64 \%$ para los SPs de $30 \mathrm{KWh}$ y $40 \mathrm{KWh}$ respectivamente. En iluminación se registró un ahorro del $40.6 \%$ para cualquiera de los valores de referencia y para el acondicionamiento de aire se registró un ahorro de $52.7 \%$ para $\mathrm{SP}=20 \mathrm{KWh}, 47,81 \%$ para $\mathrm{SP}=30 \mathrm{KWh}$ y $43.27 \%$ para un $\mathrm{SP}=40 \mathrm{KWh}$. Con respecto al consumo promedio mensual de energía eléctrica en la residencia se alcanzaron satisfactoriamente porcentajes de ahorro energético de $21.22 \%, 19.60 \%$ y $18.04 \%$ para los SPs de $20 \mathrm{KWh}, 30 \mathrm{KWh}$ y $40 \mathrm{KWh}$ respectivamente.

La automatización se comportó acorde con la ecuación elaborada del sistema. Especialmente en los días donde el consumo no es muy alto y la meta de ahorro de energía se logra cumplir, puede observarse como el sistema se reajusta para permitir niveles de comodidad más aceptables. Por otro lado, cuando el consumo de energía eléctrica es muy alto se observa que la capacidad de regulación alcanza niveles de saturación. Estas saturaciones pueden remediarse si se ajustan los valores de los tiempos del ciclo de trabajo del compresor del sistema de acondicionamiento de aire, sin embargo, esto se haría a costa de la comodidad de los inquilinos de la residencia. Un balance apropiado entre ahorro y comodidad representaría el ajuste ideal.

En la implementación de la automatización se pudo determinar que el sistema respondió conforme y congruente con el comportamiento que se observó en las simulaciones. En las gráficas presentadas en las Figuras 5 y 7 se puede apreciar desempeños similares en el comportamiento de la señal de control al igual que en la disminución del consumo de energía eléctrica para el SP seleccionado (30KWh), el cual muestra una disminución en el consumo de $55.62 \%$ en comparación con el consumo promedio de energía en ausencia de automatización. En las Figuras 6 y 8 también se puede apreciar desempeños similares en el comportamiento de la señal de control. A diferencia de la señal en la simulación en la cual se hizo uso de un set point de intensidad de iluminación de $50 \%$, en la implementación se utilizó una referencia de intensidad mínima de $60 \%$ debido a ajustes de comodidad para los inquilinos. Aún con estas diferencias se puede apreciar una reducción en el consumo de $63.01 \%$ en comparación con el consumo promedio de energía que se registró en ausencia de automatización. La red de comunicación inalámbrica basada en el protocolo Lightweight Mesh de Atmel se comportó conforme a las especificaciones [9] y resultó muy conveniente al momento de implementar la automatización debido al bajo nivel de complejidad requerido para lograr el enlace de comunicación.

\section{RECOMENDACIONES}

Dentro de un proyecto tan amplio y ambicioso como lo es el de la administración de energía eléctrica hay cabida para muchos avances y mejoras; por lo tanto, a todos aquellos investigadores interesados en este proyecto se les recomienda complementar o ampliar el sistema de gestión en hogares con redes a nivel de conjuntos residenciales; esto permitiría una gestión tentativamente más eficaz ya que permitiría una regulación sincronizada a lo largo de varios hogares, y la red de transmisión de datos inalámbrica facilita en gran medida este tipo de ampliaciones.

Esta investigación se enfocó solamente en los servicios de iluminación y climatización, sin embargo la inclusión de otros dispositivos podrían mejorar en gran medida la eficacia del sistema. Incluir electrodomésticos de alto consumo como secadoras de ropa, neveras, entre otros.

La combinación de las dos recomendaciones anteriores podría tener un impacto aún más profundo en el desempeño del sistema. Incluyendo equipos electrodomésticos de alto consumo en una red de administración de energía eléctrica automatizada en donde se podrían diseñar técnicas de rotación o alternado de los ciclos de trabajo para los servicios de alto consumo como el encendido de compresores de acondicionadores de aire o secadoras de ropa definitivamente abre las puertas a nuevos niveles en innovación de ahorro energético.

La expansión de éstos sistemas no tiene por qué limitarse a conjunto residenciales podría expandirse hasta el punto de conectar una red de administración de energía eléctrica automatizada con el proveedor de servicio, este último con una visión más amplia de la distribución de carga en todo un sector, municipio o ciudad. De manera que se pueda manejar el equilibrio de cargas a un macro nivel. El potencial de obtener ahorros significativos es enorme, sustituyendo incluso la necesidad de cortes del servicio por racionamiento. 
Es recomendable explorar el uso de modelos de control más avanzados, como el control adaptativo o redes neurales para desarrollar sistemas inteligentes que se adapten de forma automática a las costumbres y rutina de los usuarios y de esta forma capitalizar en ambos, comodidad y ahorro energético de forma totalmente transparente para el usuario.

\section{REFERENCIAS}

[1] IEA, Gadgets and Gigawatts: Policies for Energy Efficient Electronics. Paris, France, IEA, pp. 27, 2009.

[2] E. Rosas. (2010). Perspectivas del Sector Energético. [Online]. Available:

http://www.venamcham.org/index.php?view=article\&id=117\%3Aper spectivas-sector-energetico\&format=pdf\&option=com_content

[3] A. Villegas; J. Aller; P. Oliviera; M. Martínez; H. Díaz; L. Salazar; J. Molina and González, M. (2010). La USB Ante La Crisis Del Sector Eléctrico Venezolano. [Online]. Available: http://www.innovaven.org/quepasa/ecoana26.pdf

[4] W. C. Turner and S. Doty, Energy Management Handbook. Lilburn, Georgia: The Fairmont Press, 2007, pp. 577 - 578.

[5] P. G. Kini and R. C. Bansal, Energy Management Systems. Rijeka, Croatia: InTech, 2011, pp. 6.
[6] B. L. Chapeheart; W. C. Turner and W. J. Kennedy, Guide to Energy Management. Lilburn, Georgia: The Fairmont Press, 2012, pp. 37 42.

[7] S. Marttila, "Home Automation - A Challenge for Electrical Designers, Contractors and Electricians". M.S. thesis, Faculty of Electronics, Helsinki University of Technology: Espoo, Finland, 2009.

[8] A. Thumann and P.D. Metha, Handbook of Energy Engineering. Lilburn, Georgia: The Fairmont Press, 2009, pp. 279 - 302.

[9] Atmel AVR2130: Lightweight Mesh Developer Guide, Atmel Corporation, San Jose, California, pp. 4-21, 2014.

[10] Atmel AVR1631: Single Phase Energy Meter Using XMEGA A, Atmel Corporation, San Jose, California, pp. 4 - 27, 2012.

[11]8-bit Atmel XMEGA AU Microcontroller: XMEGA AU MANUAL. Atmel Corporation. San Jose, California, 2013, pp. 343.

[12]R. A. Bjørnerud, "Event System Implementations for Microcontroller Circuits". M.S. in Electronics, Norwegian University of Science and Technology, Norway. 2009.

[13] Dimming AC Incandescent Lamps Using A PIC10F200. Microchip Technology Incorporated. USA, pp. 1 - 6, 2005. 